\title{
Tomato leaf curl virus and Cotton leaf curl Multan betasatellite can cause mild transient symptoms in cotton
}

\author{
M. Saeed \\ National Institute for Biotechnology and Genetic Engineering, PO Box 577, Jhang Road, Faisalabad, Pakistan. \\ Email: saeed_hafeez@yahoo.com; msaeed@nibge.org
}

\begin{abstract}
Tomato leaf curl virus (ToLCV) from Australia is a monopartite begomovirus which is naturally associated with a DNA satellite, a vestigial betasatellite. Cotton leaf curl disease is caused by a complex consisting of one or more begomoviruses (eight species have been identified so far) associated with a single DNA $\beta$ satellite named as Cotton leaf curl Multan betasatellite (CLCuMB). ToLCV and CLCuMB caused mild symptoms in cotton plants 18-21 days postinoculation. The mild symptoms caused by ToLCV and CLCuMB in cotton plants began to diminish 6 weeks postinoculation and completely disappeared 8-10 weeks post-inoculation, raising the possibility that ToLCV may lack some factor(s) essential for persistent systemic infection of cotton.
\end{abstract}

The family Geminiviridae comprises plant-infecting, singlestranded (ss)DNA viruses. Geminiviruses cause significant crop losses throughout tropical, sub-tropical and, increasingly, temperate regions of the world. The majority of the economically important geminiviruses belong to the genus Begomovirus. Begomoviruses are transmitted by a single species of whitefly, Bemisa tabaci, and infect only dicotyledonous plants. All begomoviruses native to the New World, and a small number from the Old World, have genomes consisting of two circular ssDNA components. The majority of Old World begomoviruses have genomes consisting of a single component, homologous to the DNA A of bipartite viruses (Jeske 2009). Typical of these is Tomato leaf curl virus (ToLCV) originating from Australia (Dry et al. 1993). Most of monopartite begomoviruses, including Cotton leaf curl Multan virus (CLCuMV), have additional ssDNA satellite molecules associated with them known collectively as betasatellites. CLCuMV and its associated betasatellite (the Cotton leaf curl Multan betasatellite (CLCuMB)) are part of a begomovirus complex that has caused losses to cotton crops across Pakistan and north-western India since the late 1980s. First identified in 2000, betasatellites are a class of small circular ssDNAs $\sim 1350$ nucleotides in length. Betasatellites encode a single product, known as $\beta \mathrm{C} 1$, which is multifunctional. It is a pathogenicity determinant, a suppressor of post-transcriptional gene silencing; it raises virus DNA levels in plants and is involved in virus movement in plants (Briddon and Stanley 2006).

Tomato leaf curl virus is an Australian monopartite begomovirus which is not naturally associated with a betasatellite, although the presence of a vestigial, betasatellitederived molecule in some ToLCV infections suggests that it may have been in the past (Dry et al. 1997). Nevertheless ToLCV maintains the ability to interact with at least one betasatellite. The virus is able to trans-replicate and systemically move CLCuMB in Nicotiana tabacum (Saeed et al. 2005, 2008). The symptoms of a ToLCV infection of $N$. tabacum in the presence of CLCuMB are typical of infections of this host with CLCuMB and its cognate helper virus, CLCuMV. These symptoms are distinct from those induced by ToLCV and consist of vein swelling, vein darkening, leaf curling and the appearance of small enations on the veins on the undersides of leaves (Saeed et al. 2005, 2008).

To investigate the potential threat that ToLCV may pose to cotton cultivation in Australia, young cotton seedlings were exposed to viruliferous whiteflies reared on ToLCV-infected tomato plants. No symptoms were observed in cotton plants even at maturity, whereas all control, healthy tomato seedling showed ToLCV symptoms 15-18 days post-exposure. These results showed that by whitefly transmission ToLCV cannot cause symptoms in cotton plants (M. A. Rezaian, pers. comm.).

To determine whether ToLCV can replicate in cotton, cotton leaves were harvested from glasshouse-grown seedlings (cv. Ingard $^{\mathbb{R}}$ ) and cut into $1.5 \times 1.5 \mathrm{~cm}$ squares and kept on moistened filter paper in Petri dishes and bombarded with ToLCV and ToLCV plus CLCuMB as described previously (Saeed 2008). The leaf tissue was kept in a growth room for 3-5 days. Total nucleic acid was extracted and analysed by Southern blot hybridisation probed with ToLCV- and CLCuMB-specific probes. Southern blot analysis of DNA indicated that the replication of both ToLCV and CLCuMB was accompanied by the accumulation of both single and double-stranded replicative forms of TLCV and CLCUMB in the bombarded leaf tissues. These DNA forms were identical in size to those found in plant tissues infected with ToLCV and CLCuMB. No signal was detected in mock-inoculated cotton leaves. 

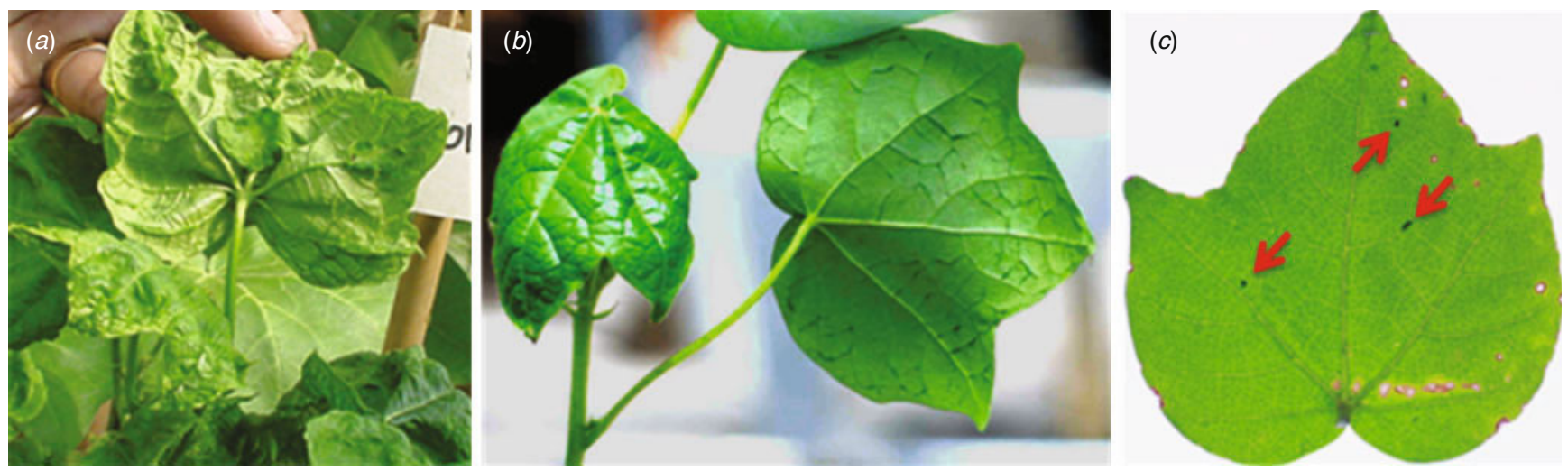

Fig. 1. A cotton plant bombarded with Cotton leaf curl Multan virus and Cotton leaf curl Multan betasatellite (CLCuMB) showing severe symptoms typical of $(a)$ cotton leaf curl diseases compared with $(b)$ a cotton plant bombarded with Tomato leaf curl virus (ToLCV) and CLCuMB showing mild symptoms. (c) The cotton leaf bombarded with ToLCV and CLCuMB showing small enation (indicated by arrows) at 6 weeks post-bombardment.

To determine whether ToLCV and CLCuMB can induce a symptomatic infection of cotton, cotton seedlings were bombarded with ToLCV and ToLCV plus CLCuMB. The control plants bombarded with CLCuMV and CLCuMB caused severe symptoms in cotton at 18-21 days postinoculation. ToLCV alone did not cause any symptom in cotton even 1 month post-inoculation. In contrast, ToLCV and CLCuMB caused mild symptoms in cotton plants 18-21 days post-inoculation (results of three independent experiments, Fig. 1). The presence of ToLCV and CLCuMB was confirmed by polymerase chain reaction (PCR) (results not shown). The mild symptoms caused by ToLCV and CLCuMB in cotton plants began to diminish 6 weeks post-inoculation (Fig. 1) and completely disappeared 8-10 weeks post-inoculation. PCR analysis was carried out to see whether newly emerging leaves of these asymptomatic cotton plants still contain the viral components. The PCR analysis on DNA extract from newly emerging leaves of these asymptomatic cotton plants could not detect ToLCV or CLCuMB (results not shown). These results indicate that although ToLCV and CLCuMB can cause mild symptoms in cotton plants, they could not sustain a systemic infection beyond 10 weeks post-inoculation; possibly ToLCV lacks some important viral sequences/factors required for sustained systemic infection of cotton.

Begomovirus as a genus is considered as emergent plant viruses (Rojas and Gilbertson 2008). Many factors are responsible for the emergent nature of begomoviruses, including: genetic changes such as random mutations, recombination and re-assortment; long-distance movement of viruses to new agro-ecosystems; changes in vector population dynamics; and acquisition of novel virus-like entities (Rojas and Gilbertson 2008). Given that ToLCV-sat DNA is a defective DNA $\beta$ (Saunders et al. 2000), it is possible that betasatellite(s) is present in weed plants in northern Australia. Tomato yellow leaf curl virus, which also has the capacity to interact with betasatellites, has been recently detected in Australia (Khan et al. 2008; Van Brunschot et al. in press). Hence pre-emptive steps need to be taken to monitor for emerging geminivirus betasatellite complex outbreaks onto cotton plants in Australia.

\section{Acknowledgements}

The author thanks Rob W. Briddon (Plant Biotechnology Division, NIBGE, Faisalabad, Pakistan) and John W. Randles (School of Agriculture, Food and Wine, University of Adelaide, Australia) for critically reading the manuscript. This project was funded by the Australian Centre for International Agricultural Research (ACIAR). M.S. thanks ACIAR and the Higher Education Commission HEC, Pakistan, for financial support.

\section{References}

Briddon RW, Stanley J (2006) Subviral agents associated with plant single-stranded DNA viruses. Virology 344, 198-210. doi:10.1016/ j.virol.2005.09.042

Dry IB, Rigden JE, Krake LR, Mullineaux PM, Rezaian MA (1993) Nucleotide sequence and genome organization of tomato leaf curl geminivirus. The Journal of General Virology 74, 147-151. doi:10.1099/0022-1317-74-1-147

Dry I, Krake LR, Rigden JE, Rezaian MA (1997) A novel subviral agent associated with a geminivirus: the first report of a DNA satellite. Proceedings of the National Academy of Sciences of the United States of America 94, 7088-7093. doi:10.1073/pnas.94.13.7088

Jeske H (2009) Geminiviruses. In 'Torque Teno Virus, Current Topics in Microbiology and Immunology'. (Eds H zur Hausen, E-M de Villiers) pp. 146-226. (Springer: Berlin)

Khan AJ, Idris AM, Al-Saady NA, Al-Mahruki MS, Al-Subhi AM, Brown JK (2008) A divergent isolate of Tomato yellow leaf curl virus from Oman with an associated DNA beta satellite: an evolutionary link between Asian and the Middle Eastern virus-satellite complexes. Virus Genes 36, 169-176. doi:10.1007/s11262-007-0163-3

Rojas MR, Gilbertson RL (2008) Emerging Plant Viruses: a Diversity of Mechanisms and Opportunities. In 'Plant Virus Evolution'. (Ed. MJ Roossinck) pp. 27-52. (Springer-Verlag: Berlin)

Saeed M (2008) Limitations observed in the use of agroinoculation for geminivirus research. Virus Genes 37, 434-435. doi:10.1007/s11262008-0279-0

Saeed M, Behjatnia SAA, Mansoor S, Zafar Y, Hasnain S, Rezaian MA (2005) A single complementary-sense transcript of a geminiviral DNA $\beta$ satellite is determinant of pathogenicity. Molecular Plant-Microbe Interactions 18, 7-14. doi:10.1094/MPMI-18-0007 
Saeed M, Mansoor S, Rezaian M, Briddon R, Randles J (2008) Satellite DNA $\beta$ overrides the pathogenicity phenotype of the $\mathrm{C} 4$ gene of Tomato leaf curl virus but does not compensate for loss of function of the coat protein and V2 genes. Archives of Virology 153, 1367-1372. doi:10.1007 s00705-008-0124-8

Saunders K, Bedford ID, Briddon RW, Markham PG, Wong SM, Stanley J (2000) A novel virus complex causes Ageratum yellow vein disease. Proceedings of the National Academy of Sciences of the United States of America 97, 6890-6895. doi:10.1073/pnas.97.12.6890
Van Brunschot SL, Persley DM, Geering ADW, Campbell PR, Thomas JE (2010) Tomato yellow leaf curl virus in Australia: distribution, detection and discovery of naturally occurring defective DNA molecules. Australasian Plant Pathology 39 (In press).

Manuscript received 13 January 2010, accepted 11 May 2010 